\title{
Non-Opioid Perioperative Pain Strategies for the Clinician: A Narrative Review
}

\author{
Alan David Kaye - Amanda L. Granier · Andrew J. Garcia • \\ Sam F. Carlson • Mitch C. Fuller · Alex R. Haroldson • \\ Shane W. White - Owen L. Krueger - Matthew B. Novitch • \\ Elyse M. Cornett
}

Received: October 10, 2019 / Published online: January 13, 2020

(C) The Author(s) 2020

\section{ABSTRACT}

Alternative and non-opioid options for pain management are necessary in perioperative patient care. Opioids are no longer touted as cure-all medications, and furthermore, there have been tremendous advances in alternative therapies such as in interventional pain, physical therapy, exercise, and nutritional counseling that have proven benefits to combat pain. The center for disease control now strongly recommends the use of multimodal analgesia and

Enhanced Digital Features To view enhanced digital features for this article go to https://doi.org/10.6084/ m9.figshare.11395071.

A. D. Kaye

Anesthesiology and Pharmacology, Toxicology, and Neurosciences, LSU School of Medicine, Shreveport, LA, USA

A. D. Kaye

Anesthesiology and Pharmacology, LSU School of Medicine, New Orleans, LA, USA

A. D. Kaye

Anesthesiology and Pharmacology, Tulane School of Medicine, New Orleans, LA, USA

A. L. Granier

LSU Health Sciences Center New Orleans, New

Orleans, LA, USA multidisciplinary approaches based on the individual needs of patients: personalized medicine. In this manuscript, the specifics of non-opioid pharmacological and non-pharmacological analgesic approaches will be discussed as well as their possible indications and uses to reduce the need for excessive use of opioids for adequate pain control.

Keywords: Alternative treatments; Chronic pain; Non-opioid; Pain management; Perioperative

\section{A. J. Garcia}

George Washington University School of Medicine and Health Sciences, Washington, DC, USA

S. F. Carlson · M. C. Fuller · A. R. Haroldson · S. W. White - O. L. Krueger

Medical College of Wisconsin, Milwaukee, WI, USA

M. B. Novitch

Department of Anesthesiology, University of Washington, Seattle, WA, USA

E. M. Cornett $(\bowtie)$

Department of Anesthesiology, LSU Health

Shreveport, Shreveport, LA, USA

e-mail: ecorne@lsuhsc.edu 


\section{Key Summary Points}

Why carry out this study?

While opioids have long been a mainstay of perioperative pain control, advances in the field of pain management have shown that exclusive use of opioids to treat pain is no longer necessary. There is a large movement toward the use of multimodal analgesia to treat pain, which has decreased opioid consumption and increased patient outcomes.

Utilizing treatments with different mechanisms may reduce the amount and length of treatment with opioids, decreasing the risk of dependence and adverse effects.

\section{What was learned from this study?}

There are many non-pharmacological treatments available that can be recommended to decrease pain.

Future research and advocacy efforts will be necessary to demonstrate the benefit of these treatments on improving patient quality of life, reducing administrative burden on providers, and saving money in the overall healthcare system.

\section{INTRODUCTION}

Perioperative pain control refers to pain control before, during, and after a surgical procedure that aims to decrease postoperative pain and increase patient outcomes [1]. Postoperative pain is an important measurable factor in perioperative pain control because it can help determine when a patient is discharged and what type of feedback a patient may give about their experience. Uncontrolled postoperative pain has a number of associated side effects including anxiety, depression, lack of sleep, and more than $80 \%$ of patients report moderate to severe postoperative pain [2]. Additionally, up to $70 \%$ of patients report significant pain even upon discharge from the hospital, suggesting that pain is still under-treated by providers [3]. Therefore, developing improved and novel treatments for treating and preventing postoperative pain is necessary.

The expansion of multimodal analgesia has been an important factor in preventing postoperative pain. Multimodal analgesia is the administration of two or more drugs with different mechanisms of action that can work together to provide pain relief [4]. The most frequently used agents in multimodal analgesia include, opioids, NMDA ( $N$-methyl-D-aspartate) antagonists (e.g., ketamine), NSAIDs (nonsteroidal anti-inflammatory drugs), epidural analgesia, regional nerve blocks, and local anesthetics (e.g., lidocaine). However, as our country continues to battle the ongoing opioid epidemic, there is a need for options other than opioids to treat pain, which we will discuss in this manuscript.

In addition to pharmacological treatments for pain management, a number of non-pharmacological preemptive modalities exist that are useful adjunct and stand-alone treatments for pain including acupuncture, massage, osteopathic manipulation, physical therapy, exercise, cognitive behavioral therapy (CBT), mindfulness meditation, and tai chi, all of which will be discussed in detail below. It is important that these treatments be acknowledged by health care providers, as there is mounting evidence suggesting their benefit [5]. Moreover, patents are more likely to follow a non-pharmacological treatment regimen if their doctor prescribes it [6]. The aim of this manuscript is to discuss non-opioid pharmacological pain management options, non-pharmacological pain management options, and the future directions in this field (see Table 1). This article is based on previously conducted studies and does not contain any studies with human participants or animals performed by any of the authors.

\section{PERIOPERATIVE PAIN TREATMENT STRATEGIES}

As previously mentioned, multimodal analgesia is the key to developing a successful 
Table 1 Pharmacological and non-pharmacological nonopioid pain treatments

\begin{tabular}{ll}
\hline Pharmacological & Non-pharmacological \\
\hline Acetaminophen & Acupuncture \\
NSAIDS & Massage \\
Antidepressants & Osteopathic manipulation \\
Anticonvulsants & Physical activity and exercise \\
Skeletal muscle & Physiotherapy and physical \\
relaxants & therapy \\
NMDA antagonists & Cognitive behavioral therapy \\
& Mindfulness meditation \\
& Tai chi \\
\hline
\end{tabular}

perioperative pain management strategy. Consistent and routine use of multimodal analgesia throughout the perioperative period can decrease the use of opioids as a primary and stand-alone treatment, and can reduce opioidrelated adverse events [4]. Furthermore, a multimodal analgesia regimen should be procedure or surgery-specific, tailored to the individual patient, and should include both pharmacological and non-pharmacological treatments. Therefore, we encourage health care providers to educate themselves about the treatments (and side effects of those treatments) that are available to use in a multimodal treatment regimen so that they can successfully employ them in their patients. And rather than asking physicians to follow a hard set rules of multimodal analgesia, we should encourage a "tool box" approach, wherein physicians are armed with a variety of knowledge and tools at their disposal, and they can choose what is best for each patient's needs.

\section{PREVENTIVE ANALGESIA}

Prevention of pain is another important component of treating perioperative pain [7]. There is much debate as to whether preventive analgesic treatment (treating pain before onset) or preemptive analgesic treatment (treating pain before a surgery) are best at decreasing postoperative pain. However, the evidence suggests that preventive analgesia reduces postoperative pain, consumption of analgesics, and seems to be the most effective at decreasing postoperative pain [8]. The overall theory explaining the limits of preemptive analgesia as a stand-alone treatment suggests that sensitization from surgical incision pain can cause a short duration of peripheral sensitization as well as a distinct central sensitization caused by initial excitation of primary afferent fibers [8]. Therefore, it seems that preventive analgesia that includes postoperative pain control (including multimodal analgesia and a longer duration of analgesic treatment) is the most effective compared to a single administration of preemptive analgesia before an incision is made. It also seems that the efficacy and duration of the preoperative analgesic itself is more important that the preoperative timing of the analgesic intervention [8]. Ideally, a preventive analgesia regimen can include a preemptive analgesic treatment along with a continued multimodal analgesic treatment. This will allow for lower doses of analgesics to be used in combination which reduces associated risks and side effects of the analgesic drugs. Ultimately this plan should be outlined by the physician and discussed in advance of the procedure with the patient.

\section{PHARMACOLOGICAL NON-OPIOID ALTERNATIVES}

\section{Acetaminophen}

Perhaps the most common and persistently used analgesic is acetaminophen, a centrally acting analgesic that has inhibitory effects on COX (cyclooxygenase) isoenzymes and serotonergic effects [9]. It can be used for mild to moderate pain in an inpatient and outpatient setting for both acute and chronic disorders and is often used perioperatively to reduce postoperative pain. While it is a dependable adjuvant and primary medication for mild to moderate pain, it also has detriments in its toxic intermediary, $\mathrm{N}$ acetyl-p-benzoquinone. Glutathione stores are used to metabolize this molecule, but once these 
stores are used up, cysteine residues can quickly damage hepatocytes. The maximum daily dose is $3000 \mathrm{mg} /$ day in healthy adults and $2000 \mathrm{mg} /$ day for those that consume more than three alcoholic beverages in a day or those that have previous liver dysfunction. In a chronic pain setting, monitoring of these limits is vital, and patients should be educated on the detrimental effects of over-ingestion [10].

\section{Nonsteroidal Anti-inflammatory Drugs (NSAIDS)}

NSAIDs inhibit COX1 and COX 2 cyclooxygenase enzymes, which results in the inhibition of prostaglandin production, decreasing the inflammatory response. As a result, this lends to mild to moderate pain reduction. There are many different classes of NSAIDs based on acid type or chemical composition, for example, ibuprofen as a propiolic acid and diclofenac as an acetic acidcontaining group. As their primary effect is antiinflammatory in nature, they do not aid in neuropathic pain. They are a preferred adjuvant or primary medication for chronic low back pain, as the etiology is usually inflammatory [11, 12]. Safety concerns exist for NSAIDs in similarity to acetaminophen; however, the organs of concern are the kidney and stomach, as they increase the risk of upper GI (gastrointestinal) bleeding, nephrotoxicity, and cardiovascular morbidity and mortality $[11,12]$. Ketorolac and indomethacin have a higher incidence of upper gastrointestinal bleeds, and the more COX2-selective NSAIDs (i.e., etodolac, celecoxib) have comparably lower risk of such a complication. The etiology of nephrotoxicity in chronic NSAID use lies in inhibition of prostaglandin-dependent renal hemodynamic perfusion, although frank nephrotic syndrome associated with these drugs has been described as well. As such, patients with stage 4 or higher chronic kidney disease (CKD) should not receive NSAIDs, and it is recommended that NSAID therapy be temporarily discontinued in those with stage 3 or higher CKD. The maximum daily dose of ibuprofen is $1200 \mathrm{mg}$, naproxen $660 \mathrm{mg}$, and aspirin $4000 \mathrm{mg}$, and are usually dosed as one pill every $4-6 \mathrm{~h}$ as needed for chronic pain.

\section{Antidepressants}

While NSAIDs and acetaminophen do not aid in neuropathic pain, antidepressants have been a mainstay of treatment of neuropathic pain disorders for years $[13,14]$. Classes include SSRIs (selective serotonin reuptake inhibitors) such as paroxetine, SNRIs (selective norepinephrine reuptake inhibitors) such as duloxetine, tricyclic antidepressants such as amitriptyline, and atypical agents such as bupropion. Each agent has a different profile based on serotonergic and norepinephrinergic effects; for example, duloxetine demonstrates a 10:1 preference for serotonergic receptors compared to norepinephrine, while milnacipran maintains a consistent 1:1 preference. It is unclear how this alters the pain management profile of these medications, but duloxetine specifically has been repeatedly shown to have positive effects on diabetic peripheral neuropathy, fibromyalgia, and chronic musculoskeletal pain [13, 14]. While these medications are exceptional for treating mild to moderate neuropathic pain, they also increase the risk for suicidal ideation, especially in those 18-24 years of age, within the first month of initiation. The most widely studied but also most concerning medication for neuropathic pain is tricyclic antidepressants. They have been shown to be effective for the treatment of neuropathic pain, but require close monitoring for adverse effects such as QTc prolongation, orthostatic hypotension, and anticholinergic symptoms [15]. Because of these side effects, they are often passed upon for SSRIs, SNRIs, and atypical agents. Typical dosages include duloxetine $60 \mathrm{mg} /$ day, paroxetine $20 \mathrm{mg} /$ day, and bupropion $150 \mathrm{mg}$ twice daily.

\section{Anticonvulsants}

Anticonvulsants contain several different classes of medications with different mechanisms of action. Gabapentinoids such as gabapentin and pregabalin inhibit calcium channels that are responsible for decreasing glutamate release. They are primarily used for diabetic peripheral neuropathy and postherpetic neuralgia [16]. Somnolence and edema are the most common 
adverse effects, and dose must be adjusted if renal function is compromised. An additional concern is abuse potential, as they cause muscle relaxation, pain reduction, and in some cases euphoria [16]. The appropriate dose varies between 1800 and $3600 \mathrm{mg} /$ day in three divided doses throughout the day.

An additional class of anticonvulsants that can be used for adjuvant therapy in chronic neuropathic pain are oxcarbazepine and carbamazepine [16]. Contrary to gabapentinoids, these agents work on voltage-gated sodium channels to decrease nerve firing. Adverse effects include drowsiness, diplopia, nausea and vomiting, and hyponatremia. Dosing varies but usually is within the 200-1000 mg maximum per day range, split into two doses, every $12 \mathrm{~h}$.

Topiramate and zonisamide are additional agents used for diabetic peripheral neuropathy and radicular low back pain, and primarily work via carbonic anhydrase inhibition [16]. This does cause concern for paresthesia, nephrolithiasis, and at high enough doses metabolic acidosis. Typical scheduling is a 4 -week titration from $25 \mathrm{mg}$ PO daily to $50 \mathrm{mg}$ PO twice daily.

\section{Skeletal Muscle Relaxants}

Skeletal muscle relaxants are used primarily for spasticity and spasms associated with musculoskeletal conditions. Medications of this type include baclofen, cyclobenzaprine, and tizanidine, and evidence for their use is limited $[17,18]$. Use of these medications often leads to very little improvement clinically and increases the risk of adverse effects such as hepatotoxicity, somnolence, and misuse $[17,18]$.

\section{NMDA Antagonists}

An interesting option for non-opioid perioperative pain are NMDA receptor antagonists, which change inhibitory pain pathways via alteration of ion channel expression, specifically postsynaptic calcium channels that are modulated by glutamate and glycine $[19,20]$. Colloquially the most common NMDA receptor modulator is ketamine, a derivative from phencyclidine with multi-receptor efficacy.
While it works primarily on NMDA receptors, it also alters opioid receptors of all types, and nicotinic and muscarinic receptors, blocks sodium and potassium channels, activates dopamine and voltage-gated calcium channels, and increases GABAA signaling [19-21].

Most recently, in July 2018, guidelines for use of ketamine in chronic pain management were published. In approximately $57 \%$ of double-blind, randomized controlled trials comparing ketamine with placebo, use of ketamine resulted in significantly reduced pain scores for non-neuropathic pain [22]. Common adverse effects associated with ketamine include sedation/somnolence, hallucinations, arrhythmias, and tachycardia. The recommendations established in July 2018 suggested that it is reasonable to start dosing with a single, outpatient infusion at a minimum dose of $80 \mathrm{mg}$ lasting more than $2 \mathrm{~h}$ and reassess before initiating further treatments [22].

\section{NON-PHARMACOLOGICAL NON- OPIOID ALTERNATIVES}

\section{Acupuncture}

Acupuncture is a traditional Chinese therapy that involves the insertion of needles into specific areas of the skin. Though acupuncture is widely used it still remains a controversial treatment. A 2018 review of acupuncture for chronic pain found that acupuncture was more effective than sham and no acupuncture treatment [23]. They also found clear evidence that the effects of acupuncture persist over time, up to 1 year after treatment. Overall, the authors concluded that acupuncture is an effective treatment for chronic pain and that decreases in pain associated with acupuncture cannot be explained solely by the placebo effect. Furthermore, a 2017 meta-analysis and review investigating the immediate analgesic effects of acupuncture for pain found that acupuncture was associated with a greater immediate pain relief effect compared to sham acupuncture and analgesic injections [24]. There is also a currently approved systematic review protocol for the identification of relevant randomized 
controlled trials involving the effectiveness of acupuncture for chronic pain with depression [25]. This study hopes to yield high-quality evidence for the relationship between chronic pain, depression, and acupuncture treatment. Although there is favorable data suggesting acupuncture as a treatment for pain, there are side effects associated with this treatment including local infection and bleeding disorders. This therapy could be used consistently leading up to the operative period in attempts to lower baseline pain levels and prepare the patient mentally for operative measures.

\section{Massage}

Therapeutic massage is the kneading or rubbing of muscles, joints, and areas of the body, usually with hands, to relieve tension and pain. Massage has been used since $2700 \mathrm{BCE}$, yet its usefulness in modern medicine is still somewhat controversial due to lack of consistent outcomes and methodological rigor in research studies. Massage is, however, often used as an adjunct therapy to analgesic treatment for pain. A 2016 systemic review and meta-analysis of randomized controlled trials of patients experiencing pain in the general population found that massage therapy, especially compared to no treatment, should be strongly recommended as an option for pain management [26]. Massage therapy was also found to be beneficial for treating anxiety and health-related quality of life. However, the study did note that the methodological differences between the studies examined made it challenging to draw sound conclusions. Another 2016 case report found that massage therapy improved nine out of 10 outcome measurements in a patient diagnosed with osteoarthritis, scoliosis, spinal stenosis, and degenerative disc disease. The most dramatic differences were in his ability to walk and in changing the degree of his pain [27]. Finally, a 2017 systematic review and meta-analysis of randomized controlled trials on the effect of massage on acute postoperative pain in critically and acutely ill adults post-thoracic surgery found that patients receiving massage with analgesia reported less pain (0-10 scale) compared to attention control/sham massage [28]. Overall, it seems that massage can be useful for treating pain, even though the methods of evaluating it in a research setting are challenging to keep consistent. More research is needed to yield sound conclusions about the relationship between pain and massage, but it can be encouraged as an adjunct treatment for pain patients.

\section{Osteopathic Manipulation}

Osteopathic manipulation is a set of hands-on techniques utilized by osteopathic physicians (DOs, or doctors of osteopathy) to diagnose, treat, and prevent illnesses and conditions. Osteopaths treat a variety of conditions and focus on manipulation of the muscles and soft tissues, while chiropractors focus on adjustments of the vertebrae and spine. A 2018 nonrandomized controlled clinical trial investigating osteopathic manipulative treatment effects on pain relief and quality of like in oncology and geriatric patients found that osteopathic manipulation produced a significant reduction in pain and a nonsignificant improvement in quality of life [29]. A 2016 randomized controlled trial investigating recovery from chronic low back pain after osteopathic manipulation found that the treatment was associated with significant measures for recovery from low back pain [30]. A 2012 randomized controlled trial of osteopathic manipulation treatment for acute low back pain in active military personnel also found support for the effectiveness of this treatment [31]. Contraindications to osteopathic manipulative therapy include deep vein thrombosis, aneurysm, frostbite, irritable or contagious skin conditions, open sores or wounds, recent surgery, recent burn, and acute flare-ups of inflammatory conditions such as rheumatoid arthritis.

\section{Physical Activity and Exercise}

Many types of pain conditions can benefit from physical activity and exercise. For many years, treatments for chronic pain included rest and inactivity. However, especially with regard to 
enhanced recovery after surgery protocols (ERAS), early mobilization and physical activity are now considered essential for recovery [32]. Furthermore, exercise can reduce pain, increase overall physical health and mental health, and improve physical functioning. A 2017 Cochrane review assessing physical activity and exercise for chronic pain in adults found that physical activity and exercise is an intervention with few adverse events that may improve pain severity and physical function, and consequent quality of life. However, due to the inconsistent methodology between studies it is difficult to make direct comparisons and yield direct outcomes [33]. A 2019 meta-analysis examining exercise-induced hypoalgesia found a positive correlation with exercise duration and analgesic effect on neck pain [34]. An interesting 2017 review article discussing whether exercise increases or decreases pain found that a single instance of fatiguing exercise in the presence of chronic pain can exacerbate that pain, however, regular exercise promotes pain relief and is associated with measurable reductions in NMDA phosphorylation, reduced serotonin transporter expression, and increase endogenous opioid system activation, all of which reduce pain [35]. Therefore, it seems that the long-term benefits of exercise are the most important to consider. In general, exercise benefits patients in their overall health and well-being and may contribute to optimizing a patient's health preoperatively depending on condition [36]. Exercise reduces pain via activation of CNS pain modulation pathways, release of beta-endorphins and neurotransmitters such as serotonin and norepinephrine, and interactions with the cardiovascular and adenosinergic systems [37].

\section{Physiotherapy and Physical Therapy}

There is some discrepancy between physiotherapy and physical therapy. Physiotherapy is considered to be a more hands-on approach manual therapy, while physical therapy indicates a more exercise-based approach to patient care. Yet some practitioners claim that the two are one in the same treatment. This discrepancy in definition also makes it difficult to discern between the two in the scientific literature where the two terms are often interchanged. That being said, a 2017 systematic review investigating the effectiveness of physiotherapy interventions for sacroiliac joint dysfunction (interestingly, published in the Journal of Physical Therapy Science) found that physiotherapy interventions are effective in the reduction of pain and disabilities associated with sacroiliac joint dysfunction, and they cited manipulation as the most effective approach [38]. A 2018 review discussing the physical therapy approaches in the treatment of low back pain found that active strategies for pain such as exercise are related to decreased disability, that no one type of exercise is more beneficial to the other (group or individual exercise), and that passive methods (rest and medications) are associated with worsening disability [39]. An important 2017 systematic review of qualitative and quantitative studies investigating physiotherapists' beliefs and attitudes on the influence of clinical practice in chronic low back pain patients found that the physiotherapist's beliefs and attitudes are important to the successful introduction of new clinical practice guidelines and models when treating patients. While this may seem like an obvious finding, it shows that the health care providers have a great deal of influence on the success of patient-oriented programs and likely the health outcomes of their patients [40]. Therefore, it is also important to consider the needs of the providers in these settings. Yet there is still work to be done. A 2019 systematic review investigating physiotherapy management of patients with chronic pelvic pain unfortunately found that the evidence currently available is sparse and contains serious methodological flaws which make it difficult to recommend a specific physiotherapy for this condition [41]. Therefore, there is still a need for studies investigating the effects of physiotherapy on specific conditions, even though most studies show that physical and physiotherapy are both beneficial for pain conditions. 


\section{Cognitive Behavioral Therapy}

With applications across psychiatric disorders of many kinds, CBT can also be applied to pain from physiological abnormalities with great efficacy. CBT is effective at managing chronic pain, either on its own or together with other pain management tools, such as meditation of pharmacological intervention. Pain is not based solely on sensory or neurologic states but is influenced by cognitive and affective processes, and the subjective manner of pain lends insight into the nonphysical aspects of the human condition. A person's thoughts and beliefs about pain can be as important as the medications or therapies they are using. CBT allows a patient to alter these thoughts and can be used throughout the preoperative, perioperative, and postoperative periods. Several studies have demonstrated the efficacy of CBT [42, 43].

There are no direct contraindications to CBT save for severe psychological disorders that require pharmacological intervention prior to discussing thoughts or experiences with a patient. There are several barriers, however, to the provision of CBT include limited access to providers, inadequate insurance coverage, lack of knowledge about CBT among health care providers, and patients' perception of stigma associated with CBT.

\section{Mindfulness Meditation}

Meditation and mindfulness can be useful to tackle the psychological components of pain that challenge patients [44]. Meditation and mindfulness often get confused, yet they are distinct concepts that can be practiced together and/or separately. Their exact definitions are often debated but they do have consistent themes. Meditation is the practice of focusing the mind to achieve calmness, stability, and clarity. Mindfulness is the practice of being fully present in the current moment. Together, mindfulness can be used during meditation to achieve calmness, stability, and clarity, by focusing on a particular object, thought or activity.
There is a growing body of literature exploring the effects of meditation on pain management. And while not all of the papers we cite here investigated pain specifically, pain was part of the patient experience and was improved upon with meditation. For example, a 2019 prospective, randomized controlled feasibility trial is currently exploring guided meditation as an adjunct to enhance postoperative recovery after cardiac surgery [45]. While pain is not the focal point of this study, postoperative pain is almost certainly associated with cardiac surgery and is an outcome measure of the study.

A 2019 integrative review of relaxation therapy with guided imagery for postoperative pain management found that this treatment was complimentary to drug analgesia [46]. A 2018 prospective, randomized, controlled study explored intraoperative meditation music as an adjunct to subarachnoid block for the improvement of postoperative outcomes following cesarean section found that patients exposed to the music has significantly less postoperative pain and anxiety and a better overall psychological well-being compared to control [47].

There is also evidence to support mindfulness as a treatment for pain. A 2010 review article summarizing peer-reviewed evidence between 1958 and 2008 for the use of relaxation and mindfulness in acute and chronic pain found that there is evidence that both relaxation and mindfulness can reduce pain outcomes in both acute and chronic pain [48]. However, an overall conclusion of the review stated that researchers and practitioners should be clearer regarding the outcomes of these studies, which due to variability between studies can be hard to compare. There is also a current randomized controlled trial exploring the effect of mindfulness training prior to total joint arthroplasty on postoperative pain and physical function; the results have not yet been published [49]. A 2019 systematic review exploring the effects of mindfulness intervention for cancer-related pain found that mindfulness-based interventions may decrease pain severity, anxiety, and depression and can improve quality of life [50]. There is also evidence to support mindfulness mediation. A 
2017 systematic review of randomized controlled trials with meta-analyses using the Hartung-Knapp-Sidik-Jonkman method for random-effects models found that mindfulness meditation is associated with a small decrease in pain compared with all types of controls [51]. A 2015 study explored the effects of mindfulness meditation on pain and quality of life of patients with low back pain and found that mindfulness-based stress reduction was effective in reduction of pain severity and the patients who practiced eight sessions of meditation reported significantly lower pain than patients who only received usual medical care [52].

Overall, more consistent research needs to be done in this area; however, the benefits of mindfulness and meditation for pain are clear. Additionally, given that every patient has unique needs, it is possible that mindfulness and meditation could make a difference for them, and therefore, should not be excluded from their treatment plan.

\section{Yoga}

Yoga is a mind-body practice that originated in India and was brought to Western culture in the nineteenth century. While the practice of yoga incorporates more than just the popularized physical postures and exercise, this type of yoga is the most widely recognized. A 2016 systematic review of the literature investigating yoga as a treatment for chronic low back pain found that yoga can reduce pain and disability, can be practiced safely in patients with chronic low back pain, and is enjoyed by participants [53]. There is also some evidence that yoga can improve physiological symptoms, but these findings are not well-established. Another 2016 systematic review investigating the effects of yoga on pain, mobility, and quality of life in patients with knee osteoarthritis found that yoga might have positive effects in relieving pain and mobility in these patients, yet the effects on the quality of life are unclear [54]. A 2017 study investigating the effects of Hatha style yoga on the treatment of pain associated with endometriosis found a reduction in the levels of chronic pelvic pain and an improvement in quality of life in participating patients
[55]. A 2015 randomized controlled pragmatic trial investigating yoga in sedentary adults with arthritis found that yoga classes can help increase physical activity and health related quality of life in this patient population [56]. Overall it seems that across a variety of pain conditions, yoga can improve both physical ability and quality of life. While there seem to be a plethora of studies investigating the effects of yoga on low back pain, there is still a need for controlled research studies investigating the effects of yoga on other pain conditions.

\section{Tai Chi}

Tai chi is an ancient Chinese tradition that is considered exercise and consists of a series of slow, focused movements performed in a focused manner along with deep breathing techniques. A 2016 systematic review and metaanalysis of randomized controlled trials investigating tai chi for chronic pain conditions found that tai chi can be considered a viable complementary and alternative medicine for chronic pain conditions and that tai chi provided immediate relief of chronic low back pain [36]. A 2018 randomized controlled pilot study investigating tai chi for older adults with chronic multisite pain showed that tai chi significantly lowered pain severity and pain interference and improved both single and dual-task gait variables, when compared to light physical exercise which did not yield these same changes [57]. A 2017 systematic review and meta-analysis investigating the effectiveness of tai chi for chronic musculoskeletal pain conditions had ambiguous findings, concluding that tai chi was only moderately more effective compared to no treatment and suggested that more high-quality trials with larger sample sizes are necessary to yield sound conclusions [58]. Interestingly, tai chi along with probiotics and nutrition improvement have all been suggested to be valuable changes in lifestyle that can improve quality of life in patients with chronic pain. A 2012 meta-analysis of all three suggested lifestyle changes found considerable improvement of symptoms, especially in patients doing qi gong, tai chi, and yoga [59]. Therefore, similar to the previously mentioned alternative 
treatments, there is evidence to suggest that tai chi is beneficial for some pain patients, but more research is needed before definitive conclusions can be drawn.

\section{Locus of Control}

Locus of control is a phenomenon in personality psychology that is defined by the degree to which people believe they have control over their health, outcomes, and life events. A strong internal locus of control is associated with an individual believing that the events in their life are derived primarily from their own actions. A strong external locus of control is associated with an individual believing that life events are out of their control. Health locus of control is associated with health outcomes related to the internal or external type. A 2018 study explored the effects of health locus of control in pain intensity outcomes on conservatively and operatively treated hand surgery patients. They found that patients suffering greater initial pain intensity who also had low health locus of control experienced a less favorable treatment outcome. They further extrapolated that if these patients succeed in transferring perceived health control to professionals and gained confidence in treatment and clinicians, that their treatment outcomes could have been improved [60]. A 2012 cross-sectional study found that patients in treatment for chronic lower back pain have higher externalized beliefs, suggesting that these patients believe their pain was related to events that were out of their control [61]. A 2017 cross-sectional questionnaire study explored the role of health locus of control in evaluating depression in patients with chronic pain conditions and found that patients with an internal locus of control are less depressed compared to patients with fatalistic views on their health and pain [62]. These studies are important because they suggest that the patients mental outlook can affect their health outcomes. Ideally, health care providers could add a series of questions to their patient health background information that could help them understand the mental outlook of their patients. If their patient has an external locus of control perhaps the health care provider can change their approach to help the patient understand and trust them more. This would be an extension of an individualized health treatment strategy tailored to each patient.

\section{FUTURE DIRECTIONS}

There is a push to recategorize pain beyond descriptors based on a particular diagnosis, such as nociceptive and neuropathic pain, towards a greater focus on the identification of pain phenotypes based on qualities of pain such as burning or stabbing [63]. Identification of such phenotypes will allow clinicians and researchers to better address underlying neural pathways mediating pain. Additionally, advances in understanding of signaling pathways, receptors, and pain pathophysiology are identifying several novel targets that may lead to more effective pain treatments.

Several emerging non-opioid treatments for acute and chronic pain are in phase 2 and 3 trials as of the writing of this article. Among those attracting intense interest include those targeting voltage-gated calcium channels, particularly isoforms $1.7,1.8$, and $1.9[63,64]$. The first candidate targeting the Nav1.7 channel, designated CNV1014802, has even finished phase 2 studies as of 2019 [65]. Other novel agents actively undergoing trials include monoclonal antibodies targeting IL-6 and nerve growth factor (NGF), as well as allogeneic cartilage cell replacement therapy. Additionally, various clinical trials are studying novel formulations of existing treatments such as cyclobenzaprine and clonidine. Lastly, basic research is underway investigating PGE1 synthase and cannabinoid targets, while targets such as tetrahydrobiopterin (BH4), purinoceptors implicated in neuropathic and inflammatory pain pathways, potassium-channel openers, and store-operated calcium channels are emerging as future potential targets [64].

The delivery and reimbursement of novel care strategies also face their own unique challenges. The latest guidelines for opioid prescribing from the Centers for Disease Control and Prevention (CDC) in 2016 note that most 
studies for proof of efficacy with opioids are based on short-term rather than chronic pain [66]. Doctors are encouraged to prescribe nondrug treatments, such as physical therapy, first and then set realistic goals for reducing pain with an emphasis on improved functioning, rather than elimination of pain. Multi-modal approaches, including talk, swim, and physical therapy, meditation, hypnosis, yoga, and biofeedback, have shown synergistic benefit when used in addition to medication for pain treatment. One study has even shown that using non-pharmacological, non-opioid pain reduction strategies in the inpatient postoperative setting resulted in cost savings of $\$ 898$ per admission to the health system, illustrating a cost-benefit for providing multi-modal options for pain control [67].

\section{CONCLUSION}

In conclusion, perioperative pain control utilizing multimodal analgesia without opioids is a useful treatment strategy that is gaining traction. Physicians should educate themselves on novel pharmacological and non-pharmacological treatments as they become available and should actively use these treatments for their patients. There is growing evidence suggesting that while opioids have an important role in treating perioperative pain, they certainly should not be the primary or the stand-alone treatment for pain. Patients will be more likely to embrace these new options if their health care providers take an active role in prescribing these treatments and tailoring these treatments to the individual patient.

\section{ACKNOWLEDGEMENTS}

Funding. No funding or sponsorship was received for this study or publication of this article.

Authorship. All named authors meet the International Committee of Medical Journal Editors (ICMJE) criteria for authorship for this article, take responsibility for the integrity of the work as a whole, and have given their approval for this version to be published.

Disclosures. Amanda L. Granier, Andrew J. Garcia, Sam Carlson, Mitch Fuller, Alex Haroldson, Shane White, Owen Krueger, Matthew Novitch, and Elyse M. Cornett have no disclosures to state. Alan David Kaye is a member of the journal's Editorial Board.

Compliance with Ethics Guidelines. This article is based on previously conducted studies and does not contain any studies with human participants or animals performed by any of the authors.

Open Access. This article is distributed under the terms of the Creative Commons Attribution-NonCommercial 4.0 International License (http://creativecommons.org/licenses/ by-nc/4.0/), which permits any noncommercial use, distribution, and reproduction in any medium, provided you give appropriate credit to the original author(s) and the source, provide a link to the Creative Commons license, and indicate if changes were made.

\section{REFERENCES}

1. Cohen MJ, Schecter WP. Perioperative pain control: a strategy for management. Surg Clin N Am [Internet]. 2005;85(6):1243-57. https://linkinghub. elsevier.com/retrieve/pii/S0039610905001246. Accessed 10 Sept 2019.

2. Sinatra R. Causes and consequences of inadequate management of acute pain. Pain Med. 2010;11(12): 1859-71.

3. Apfelbaum JL, Chen C, Mehta SS, Gan TJ. Postoperative pain experience: results from a national survey suggest postoperative pain continues to be undermanaged. Anesth Analg. 2003;97(2):534-40 (table of contents).

4. Schwenk ES, Mariano ER. Designing the ideal perioperative pain management plan starts with multimodal analgesia. Korean J Anesthesiol [Internet]. 2018;71(5):345-52. http://ekja.org/journal/view. php?doi=10.4097/kja.d.18.00217. Accessed 10 Sept 2019. 
5. Gannotta R, Malik S, Chan AY, Urgun K, Hsu F, Vadera S. Integrative medicine as a vital component of patient care. Cureus [Internet]. 2018;10(8): e3098. http://www.ncbi.nlm.nih.gov/pubmed/ 30338174. Accessed 9 Sept 2019.

6. Loftus AM, McCauley F, McCarron MO. Impact of social prescribing on general practice workload and polypharmacy. Public Health [Internet]. 2017;148: 96-101. https://linkinghub.elsevier.com/retrieve/ pii/S0033350617301270. Accessed 9 Sept 2019.

7. Sittl R, Irnich D, Lang PM. Update zur präemptiven Analgesie. Anaesthesist. 2013;62(10):789-96.

8. Vadivelu N, Mitra S, Schermer E, Kodumudi V, Kaye $\mathrm{AD}$, Urman RD. Preventive analgesia for postoperative pain control: a broader concept. Local Reg Anesth [Internet]. 2014;7:17. http://www.ncbi.nlm. nih.gov/pubmed/24872720. Accessed 18 Sept 2019.

9. Cooper TE, Fisher E, Anderson B, Wilkinson NM, Williams DG, Eccleston C. Paracetamol (acetaminophen) for chronic non-cancer pain in children and adolescents. Cochrane Database Syst Rev. 2017;8:CD012539.

10. Ennis ZN, Dideriksen D, Vaegter HB, Handberg G, Pottegård A. Acetaminophen for chronic pain: a systematic review on efficacy. Basic Clin Pharmacol Toxicol. 2016;118(3):184-9. https://doi.org/10. 1111/bcpt.12527.

11. Eccleston C, Cooper TE, Fisher E, Anderson B, Wilkinson NM. Non-steroidal anti-inflammatory drugs (NSAIDs) for chronic non-cancer pain in children and adolescents. Cochrane Database Syst Rev. 2017;8:CD012537.

12. Ho KY, Gwee KA, Cheng YK, Yoon KH, Hee HT, Omar AR. Nonsteroidal anti-inflammatory drugs in chronic pain: implications of new data for clinical practice. J Pain Res. 2018;11:1937-48.

13. Patetsos E, Horjales-Araujo E. Treating chronic pain with SSRIs: what do we know? Pain Res Manag. 2016;2016:2020915.

14. Riediger C, Schuster T, Barlinn K, Maier S, Weitz J, Siepmann T. Adverse effects of antidepressants for chronic pain: a systematic review and meta-analysis. Front Neurol. 2017;8:307.

15. Sansone RA, Sansone LA. Pain, pain, go away: antidepressants and pain management. Psychiatry (Edgmont). 2008;5(12):16-9.

16. Sidhu HS, Sadhotra A. Current status of the new antiepileptic drugs in chronic pain. Front Pharmacol. 2016;7:276.
17. Park HJ, Moon DE. Pharmacologic management of chronic pain. Korean J Pain. 2010;23(2):99-108.

18. Ottawa (ON): Canadian Agency for Drugs and Technologies in Health. Long-term use of cyclobenzaprine for pain: a review of the clinical effectiveness [Internet]. CADTH Rapid Response Reports. 2015. https://www.ncbi.nlm.nih.gov/ pubmed/25763449.

19. Littlejohn G, Guymer E. Modulation of NMDA receptor activity in fibromyalgia. Biomedicines. 2017;5(2):15.

20. Hewitt DJ. The use of NMDA-receptor antagonists in the treatment of chronic pain. Clin J Pain. 2000;16(2 Suppl):S73-9.

21. New Ketamine Guidelines for Acute and Chronic Pain Published-American Society of Regional Anesthesia and Pain Medicine. https://www.asra. com/news/200/new-ketamine-guidelines-for-acuteand-ch.

22. Cohen SP, Bhatia A, Buvanendran A, Schwenk ES, Wasan AD, Hurley RW, et al. Consensus Guidelines on the Use of Intravenous Ketamine Infusions for Chronic Pain From the American Society of Regional Anesthesia and Pain Medicine, the American Academy of Pain Medicine, and the American Society of Anesthesiologists. Reg Anesth Pain Med. 2018;43(5):1.

23. Vickers AJ, Vertosick EA, Lewith G, MacPherson H, Foster NE, Sherman KJ, et al. Acupuncture for chronic pain: update of an individual patient data meta-analysis. J Pain [Internet]. 2018;19(5):455-74. http://www.ncbi.nlm.nih.gov/pubmed/29198932. Accessed 2 Oct 2019.

24. Xiang A, Cheng K, Shen X, Xu P, Liu S. The immediate analgesic effect of acupuncture for pain: a systematic review and meta-analysis. Evid Based Complement Alternat Med [Internet]. 2017;2017: 3837194. http://www.ncbi.nlm.nih.gov/pubmed/ 29234385. Accessed 2 Oct 2019.

25. Yang Z, Zhao L, Xie X, Xu T, Zhang Y, Wang X, et al. The effectiveness of acupuncture for chronic pain with depression: a systematic review protocol. Medicine (Baltimore) [Internet]. 2017;96(47): e8800. http://www.ncbi.nlm.nih.gov/pubmed/ 29381981. Accessed 2 Oct 2019.

26. Crawford C, Boyd C, Paat CF, Price A, Xenakis L, Yang E, et al. The impact of massage therapy on function in pain populations-a systematic review and meta-analysis of randomized controlled trials: part I, patients experiencing pain in the general population. Pain Med. 2016;17(7):1353-75. 
27. Allen L. Case study: the use of massage therapy to relieve chronic low-back pain. Int J Ther Massage Bodyw [Internet]. 2016;9(3):27-30. http://www. ncbi.nlm.nih.gov/pubmed/27648110. Accessed 18 Sept 2019.

28. Boitor M, Gélinas C, Richard-Lalonde M, Thombs BD. The effect of massage on acute postoperative pain in critically and acutely ill adults post-thoracic surgery: systematic review and meta-analysis of randomized controlled trials. Hear Lung [Internet]. 2017;46(5):339-46. http://www.ncbi.nlm.nih.gov/ pubmed/28619390. Accessed 18 Sept 2019.

29. Arienti C, Bosisio T, Ratti S, Miglioli R, Negrini S. Osteopathic manipulative treatment effect on pain relief and quality of life in oncology geriatric patients: a nonrandomized controlled clinical trial. Integr Cancer Ther. 2018;17(4):1163-71.

30. Licciardone JC, Gatchel RJ, Aryal S. Recovery from chronic low back pain after osteopathic manipulative treatment: a randomized controlled trial. J Am Osteopath Assoc [Internet]. 2016;116(3):144. http://www.ncbi.nlm.nih.gov/pubmed/26927908. Accessed 7 Oct 2019.

31. Cruser des A, Maurer D, Hensel K, Brown SK, White K, Stoll ST. A randomized, controlled trial of osteopathic manipulative treatment for acute low back pain in active duty military personnel. J Man Manip Ther [Internet]. 2012;20(1):5-15. http:// www.ncbi.nlm.nih.gov/pubmed/23372389. Accessed 7 Oct 2019.

32. Beverly A, Kaye AD, Ljungqvist O, Urman RD. Essential elements of multimodal analgesia in enhanced recovery after surgery (ERAS) guidelines. Anesthesiol Clin [Internet]. 2017;35(2):e115-43. http://www.ncbi.nlm.nih.gov/pubmed/28526156. Accessed 13 Nov 2017.

33. Geneen LJ, Moore RA, Clarke C, Martin D, Colvin LA, Smith BH. Physical activity and exercise for chronic pain in adults: an overview of cochrane reviews. Cochrane database Syst Rev [Internet]. 2017;4(4):CD011279. http://www.ncbi.nlm.nih. gov/pubmed/28436583. Accessed 7 Oct 2019.

34. Polaski AM, Phelps AL, Kostek MC, Szucs KA, Kolber BJ. Exercise-induced hypoalgesia: a meta-analysis of exercise dosing for the treatment of chronic pain. PLoS One [Internet]. 2019;14(1):e0210418. http:// www.ncbi.nlm.nih.gov/pubmed/30625201. Accessed 7 Oct 2019.

35. Lima L V, Abner TSS, Sluka KA. Does exercise increase or decrease pain? Central mechanisms underlying these two phenomena. J Physiol [Internet]. 2017;595(13):4141-50. http://www.ncbi.nlm. nih.gov/pubmed/28369946. Accessed 7 Oct 2019.
36. Kong LJ, Lauche R, Klose P, Bu JH, Yang XC, Guo $\mathrm{CQ}$, et al. Tai Chi for chronic pain conditions: a systematic review and meta-analysis of randomized controlled trials. Sci Rep. 2016;6:25325.

37. Martins DF, Mazzardo-Martins L, Soldi F, Stramosk J, Piovezan AP, Santos ARS. High-intensity swimming exercise reduces neuropathic pain in an animal model of complex regional pain syndrome type I: evidence for a role of the adenosinergic system. Neuroscience. 2013;234:69-76.

38. Al-Subahi M, Alayat M, Alshehri MA, Helal O, Alhasan $\mathrm{H}$, Alalawi A, et al. The effectiveness of physiotherapy interventions for sacroiliac joint dysfunction: a systematic review. J Phys Ther Sci [Internet]. 2017;29(9):1689-94. http://www.ncbi. nlm.nih.gov/pubmed/28932014. Accessed 7 Oct 2019.

39. Shipton EA. Physical therapy approaches in the treatment of low back pain. Pain Ther [Internet]. 2018;7(2):127-37. http://www.ncbi.nlm.nih.gov/ pubmed/30229473. Accessed 7 Oct 2019.

40. Gardner T, Refshauge K, Smith L, McAuley J, Hübscher M, Goodall S. Physiotherapists' beliefs and attitudes influence clinical practice in chronic low back pain: a systematic review of quantitative and qualitative studies. $\mathrm{J}$ Physiother [Internet]. 2017;63(3):132-43. http://www.ncbi.nlm.nih.gov/ pubmed/28655562. Accessed 7 Oct 2019.

41. Klotz SGR, Schön M, Ketels G, Löwe B, Brünahl CA. Physiotherapy management of patients with chronic pelvic pain (CPP): a systematic review. Physiother Theory Pract [Internet]. 2019;35(6): 516-32. http://www.ncbi.nlm.nih.gov/pubmed/ 29589778. Accessed 7 Oct 2019.

42. Lim J-A, Choi S-H, Lee WJ, Jang JH, Moon JY, Kim YC, et al. Cognitive-behavioral therapy for patients with chronic pain: implications of gender differences in empathy. Medicine (Baltimore). 2018;97(23):e10867.

43. Knoerl R, Lavoie Smith EM, Weisberg J. Chronic pain and cognitive behavioral therapy. West J Nurs Res. 2016;38(5):596-628.

44. Zeidan F, Vago DR. Mindfulness meditation-based pain relief: a mechanistic account. Ann N Y Acad Sci [Internet]. 2016;1373(1):114-27. http://www. ncbi.nlm.nih.gov/pubmed/27398643. Accessed 10 Sept 2019.

45. Packiasabapathy S, Susheela AT, Mueller A, Patxot M, Gasangwa D-V, O'Gara B, et al. Guided meditation as an adjunct to enhance postoperative recovery after cardiac surgery: study protocol for a prospective randomized controlled feasibility trial. Trials [Internet]. 2019;20(1):39. http://www.ncbi. 
nlm.nih.gov/pubmed/30635064. Accessed 10 Sept 2019.

46. Felix MM dos S, Ferreira MBG, da Cruz LF, Barbosa $\mathrm{MH}$. Relaxation therapy with guided imagery for postoperative pain management: an integrative review. Pain Manag Nurs [Internet]. 2019;20(1):3-9. http://www.ncbi.nlm.nih.gov/pubmed/29249618. Accessed 10 Sept 2019.

47. Kurdi MS, Gasti V. Intraoperative meditation music as an adjunct to subarachnoid block for the improvement of postoperative outcomes following cesarean section: a randomized placebo-controlled comparative study. Anesth Essays Res [Internet]. 2018;12(3):618-24. http://www.ncbi.nlm.nih.gov/ pubmed/30283165. Accessed 10 Sept 2019.

48. Dunford E, DClinPsy MT. Relaxation and mindfulness in pain: a review. Rev pain [Internet]. 2010;4(1):18-22. http://www.ncbi.nlm.nih.gov/ pubmed/26524978. Accessed 10 Sept 2019.

49. Dowsey MM, Castle DJ, Knowles SR, Monshat K, Salzberg MR, Choong PFM. The effect of mindfulness training prior to total joint arthroplasty on post-operative pain and physical function: study protocol for a randomised controlled trial. Trials [Internet]. 2014;15:208. http://www.ncbi.nlm.nih. gov/pubmed/24899242. Accessed 11 Sept 2019.

50. Ngamkham S, Holden JE, Smith EL. A systematic review: mindfulness intervention for cancer-related pain. Asia-Pacific J Oncol Nurs [Internet]. 2019;6(2): 161-9. http://www.ncbi.nlm.nih.gov/pubmed/ 30931361. Accessed 11 Sept 2019.

51. Hilton L, Hempel S, Ewing BA, Apaydin E, Xenakis L, Newberry S, et al. Mindfulness meditation for chronic pain: systematic review and meta-analysis. Ann Behav Med. 2017;51(2):199-213.

52. Banth S, Ardebil MD. Effectiveness of mindfulness meditation on pain and quality of life of patients with chronic low back pain. Int J Yoga [Internet]. 2015;8(2):128-33. http://www.ncbi.nlm.nih.gov/ pubmed/26170592. Accessed 11 Sept 2019.

53. Chang DG, Holt JA, Sklar M, Groessl EJ. Yoga as a treatment for chronic low back pain: a systematic review of the literature. J Orthop Rheumatol [Internet]. 2016;3(1):1-8. http://www.ncbi.nlm.nih. gov/pubmed/27231715. Accessed 7 Oct 2019.

54. Kan L, Zhang J, Yang Y, Wang P. The effects of yoga on pain, mobility, and quality of life in patients with knee osteoarthritis: a systematic review. Evid Based Complement Alternat Med [Internet]. 2016;2016:6016532. http://www.ncbi.nlm.nih.gov/ pubmed/27777597. Accessed 7 Oct 2019.
55. Gonçalves AV, Barros NF, Bahamondes L. The practice of Hatha yoga for the treatment of pain associated with endometriosis. J Altern Complement Med [Internet]. 2017;23(1):45-52. http:// www.ncbi.nlm.nih.gov/pubmed/27869485. Accessed 7 Oct 2019.

56. Moonaz SH, Bingham CO, Wissow L, Bartlett SJ, Bartlett SJ. Yoga in sedentary adults with arthritis: effects of a randomized controlled pragmatic trial. J Rheumatol [Internet]. 2015;42(7):1194-202. http://www.ncbi.nlm.nih.gov/pubmed/25834206. Accessed 7 Oct 2019.

57. You T, Ogawa EF, Thapa S, Cai Y, Zhang H, Nagae S, et al. Tai Chi for older adults with chronic multisite pain: a randomized controlled pilot study. Aging Clin Exp Res [Internet]. 2018;30(11):1335-43. http://www.ncbi.nlm.nih.gov/pubmed/29512041. Accessed 7 Oct 2019.

58. Hall A, Copsey B, Richmond H, Thompson J, Ferreira M, Latimer J, et al. Effectiveness of Tai Chi for chronic musculoskeletal pain conditions: updated systematic review and meta-analysis. Phys Ther [Internet]. 2017;97(2):227-38. http://www.ncbi. nlm.nih.gov/pubmed/27634919. Accessed 7 Oct 2019.

59. Langhorst J, Häuser W, Bernardy K, Lucius H, Settan $\mathrm{M}$, Winkelmann $\mathrm{A}$, et al. Komplementäre und alternative Verfahren beim Fibromyalgiesyndrom. Der Schmerz. 2012;26(3):311-7.

60. Stewart JA, Aebischer V, Egloff N, Wegmann B, von Känel R, Vögelin E, et al. The role of health locus of control in pain intensity outcome of conservatively and operatively treated hand surgery patients. Int J Behav Med [Internet]. 2018;25(3):374-9. http:// www.ncbi.nlm.nih.gov/pubmed/29488207. Accessed 11 Sept 2019.

61. Oliveira TH, Oliveira VC, Melo RC, Melo RM, Freitas AE, Ferreira PH. Patients in treatment for chronic low back pain have higher externalised beliefs: a cross-sectional study. Rev Bras Fisioter [Internet]. 16(1):35-9. http://www.ncbi.nlm.nih. gov/pubmed/22441226. Accessed 11 Sept 2019.

62. Wong HJ, Anitescu M. The role of health locus of control in evaluating depression and other comorbidities in patients with chronic pain conditions, a cross-sectional study. Pain Pract [Internet]. 2017;17(1):52-61. http://www.ncbi.nlm.nih.gov/ pubmed/26895696. Accessed 11 Sept 2019.

63. Worley SL. New directions in the treatment of chronic pain: national pain strategy will guide prevention, management, and research. Pharm Ther. 2016;41(2):107-14. 
64. Bonnie RJ, Ford MA PJ, editor. Progress and future directions in research on pain and opioid use disorder. pain management and the opioid epidemic: balancing societal and individual benefits and risks of prescription opioid use. Washington DC: The National Academies Press;.

65. Pain Management and the Opioid Epidemic: Balancing Societal and Individual Benefits and Risks of Prescription Opioid Use. Progress and future directions in research on pain and opioid use disorder, chap 3, pp. 119-184. 2017.

66. America's Pain: The Opioid Epidemic. 2018.

67. Dusek JA, Griffin KH, Finch MD, Rivard RL, Watson D. Cost savings from reducing pain through the delivery of integrative medicine program to hospitalized patients. J Altern Complement Med. 2018;24(6):557-63. 